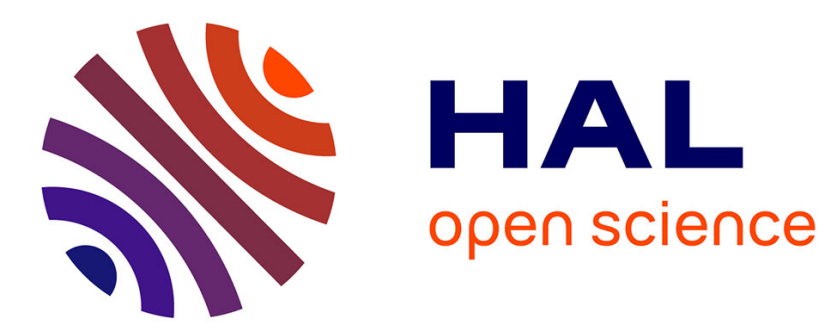

\title{
Powerline Communication System for Monitoring and Supervision of Feeder Equipments for MV Substation Automation
}

Liping Lu, Gangyan Li, Ye-Qiong Song

\section{- To cite this version:}

Liping Lu, Gangyan Li, Ye-Qiong Song. Powerline Communication System for Monitoring and Supervision of Feeder Equipments for MV Substation Automation. IEEE Symposium on Industrial Embedded Systems, Oct 2006, Antibes Juan-Les-Pins, France. inria-00113787

\section{HAL Id: inria-00113787 \\ https://hal.inria.fr/inria-00113787}

Submitted on 14 Nov 2006

HAL is a multi-disciplinary open access archive for the deposit and dissemination of scientific research documents, whether they are published or not. The documents may come from teaching and research institutions in France or abroad, or from public or private research centers.
L'archive ouverte pluridisciplinaire HAL, est destinée au dépôt et à la diffusion de documents scientifiques de niveau recherche, publiés ou non, émanant des établissements d'enseignement et de recherche français ou étrangers, des laboratoires publics ou privés. 


\title{
Powerline Communication System for Monitoring and Supervision of Feeder Equipments for MV Substation Automation
}

\author{
LiPing $\mathrm{LU}^{1,2}$, GangYan $\mathrm{LI}^{1}$, YeQiong $\mathrm{SONG}^{2}$ \\ 1 - School of Mechanical and Electronical \\ engineering, Wuhan university of technology, \\ 430070 Wuhan, Hubei, P.R. China \\ gangyanli@whut.edu.cn \\ 2 - LORIA-INPL, Campus Scientique-BP239, \\ 54506 Vandoeuvre-lès-Nancy-France \\ \{lu, song\}@loria.fr
}

\begin{abstract}
With the deregulation of power market and for providing better services to electric consumers, the current substation should be automated. In the past decade, new communications schemes have been designed and retrofitted into the substations by the utilities to integrate data from relays and Intelligent Electronic Devices (IEDs) and capitalize on the protection, control, metering, fault recording, communication functions available in digital devices. Generally, using station bus to connect all equipments inside the substation, except some primary equipments which are outsider substations such as MV/LV transformer and switchgears. For those outsider equipments, we propose using the REMPLI powerline communication (PLC) technologies to communicate with the substation. The simulation results have shown that the REMPLI PLC network guarantees the substation automation requirements.
\end{abstract}

\section{Introduction}

Electric power industry has confronted many new challenges in the deregulated environment. There has been increased pressure on the electricity utilities to utilize network assets more effectively and provide a reliable and high quality power supply. The distribution network provides the final link between the bulk transmission system and the customers. It has been reported that $80 \%$ of the customer service interruptions are due to failures in the distribution networks. In order to improve service reliability, the existing substation should be automated for faster fault location and clearance, cooperating with the feeder automation. And in the past decade, new communications schemes have been designed and retrofitted into the substations by the utilities to integrate data from relays and Intelligent Electronic Devices (IEDs) and capitalize on the protection, control, metering, fault recording, communication functions available in digital devices [3].

Substation automation is defined as deployment of substation and feeder operating functions and applications ranging from SCADA and alarm processing to integrated volt/var control in order to optimize the management of capital assets and enhance operation and maintenance (O\&M) efficiencies with minimal human intervention [2].

Generally, station bus is used to connect all equipments inside the substation. Since MV substation control system often controls MV network objects (feeder automation), it has to be able to control part of equipment locally and part of equipment remotely at the same time. Such remote objects are MV/LV Transformer Stations for transformation from $\mathrm{MV}$ to $\mathrm{LV}$, pole-tops with reclosers using Circuit Breakers and with remotely controlled disconnectors [1]. For control of MV network objects outside the substations, whatever communication facilities available should be used.

The construction of private wired network costs much, since the MV feeder equipments are distributed widely. And there are difficulties to access to certain equipments using wireless technology since those equipments are often located in closed environments with metallic obstacles (reinforced concrete walls and tubes) and with strong EMI (Electromagnetic Interference). So it is ideal to use the power line as a communication medium to construct an economic, secure and reliable communication system, because no new wires are needed for the low cost, the reliable and high transmission speed PLC chips are available for the efficiency, and power line is owned by the distribution utility for the security.

This paper is structured as follows:

First, a short overview on the communication requirements for outsider substation system is presented in section 2. The relevant characteristics of the PLC are explained and a suitable architecture for the outsider substation system is described in section 3. Section 4 recall REMPLI communication protocols that we developed [5] $[6][7][8]$. Simulation results are given in section 5. Finally a brief conclusion can be found in section 6 .

\section{Requirements for outsider substation communication system}

Some substation automation functions need the Substation IEDs to communicate with external IEDs. Those functions include [2]:

--Implementation of Fault Location, Fault Isolation and 
Service Restoration functionality which may require Substation IEDs to communicate with external IEDs for switches, reclosers, or sectionalizers.

--Implementation of Voltage Dispatch on the distribution system which requires communications between substation IEDs and feeder IEDs. These communications are used for coordinating operation of power equipment devices on the substation with those located along distribution feeders served by the substation.

--VAR Dispatch requires low-speed communications between substation IEDs and IEDs located on the distribution system. These communications are used for coordinating operation of substation and feeder devices to optimize the power factor along the distribution feeder.

Application-to-application delay (or end-to-end delay) is defined as the sum of the times required for the sending IED communication processor to accept the data from the sending application "fl", and exit the output queue of the sender "a", plus time over the communication network "b", plus the time "c" required for the receiving IED communication processor to extract the message content and present it to the receiving application "f 2 ". As a matter of fact, only time "b" is the network delay, which includes the processing time required by routers, bridges, gateways, etc. Figure 1 shows time components that define the time requirement.

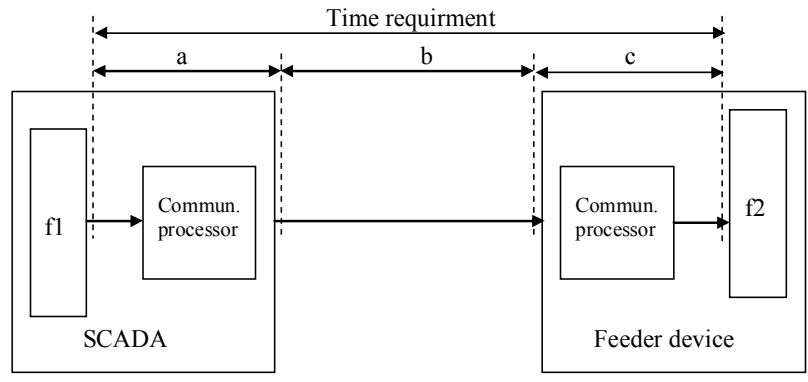
Figure 1. Application-to-Application
communication times

The main application specific communication performance requirements are shown in the Table I. All the applications are aperiodic tasks. The SCADA estimates the distribution network conditions according the data collected at insider substation and makes some decisions to send commands to the feeder devices. "Maximum Delivery Time" defines a range, or the qualifier "up to", for the Application-to-application time requirements.

For giving the performance constraints of the communication network, we assume that the processor time is a very small duration which is not necessary to involve into the value "a", "b", "c". Generally, the SCADA server is connected with the inside substation bus which uses a gateway to connect the outsider substation communication network. So the communication time has four parts: medium access delay and transmission time for insider and outsider communication network, respectively. The incorporation of high speed Ethernet (100 Mbps -1 Gbps) as a future communication backbone both inside a substation and between substations and control centers appears to be an accepted fact [1]. With the industrial Ethernet widely applied in the industrial automation, insider substation network transmission time and access delay of high priority is indeed small. For the simplification, we can roughly consider the Table I as outsider substation communication performance constraints.

\section{Table I}

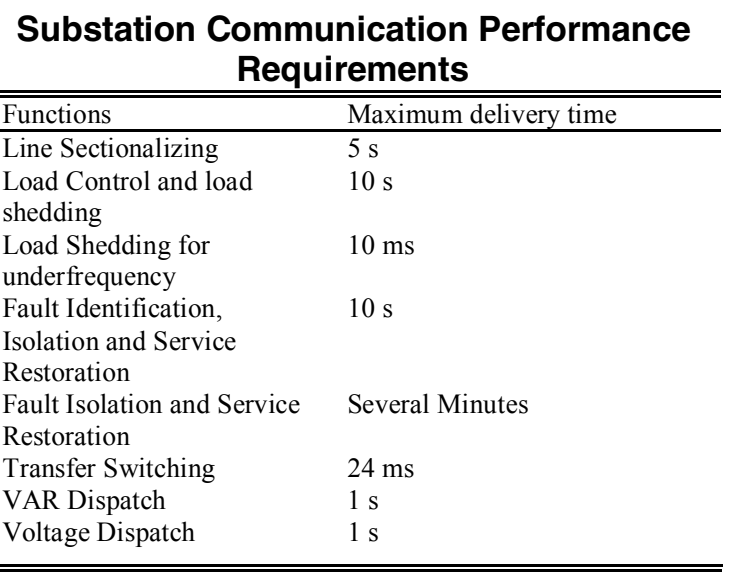

We conclude that the most application communication time constraints are more than 1 second; only two have very small transmission time.

\section{Powerline communication and system architecture}

Substation automation requires data transfer between several remote devices and substation. The SCADA service in the substation monitors and controls the remote devices. Traditionally no peer-to-peer communication being required, a master-slave architecture is appropriate. Using the power line as data links between substations and IEDs, feeder data are collected and gathered at the substation data concentrators (RTU). The possible architecture for substation automation is shown in Figure 2. The PLC network roughly follows the topology of the distribution grid. It consists of master station (RTU) located in the substation and one or several slave stations (IEDs). The master station controls slave stations for accessing the communication medium. This control reaches from governing medium access to configuration, e.g. routing and repeater management or maximum polling time.

Since powerline is designed to deliver the power, not for the communication, it presents a hostile communication environment. In fact, PLC physical layer offers highly variable characteristics due to the time-variable noises injected by electrical devices. It shows a time-varying transmission bit rate. It is not easy to guarantee a certain bandwidth and maximum transfer delay. Moreover, in a wide area PLC network, transmitting a packet from a source to a not immediately reachable destination node requires the packet relay of the intermediate nodes (repeaters). However, 
considering the dynamic topology change and impossible prediction of the powerline attenuation, repeaters cannot be statically configured.

REMPLI ${ }^{1}$ (Real-time Energy Management via Power Lines and Internet) system uses Medium Voltage (MV) and Low Voltage (LV) power grid as communication media to implements wide-area control and monitoring, customer metering reading. It means that communication is not bound to a serial line that establishes a point-to-point connection between a field device and an application, but consists of different network segments, which have to communicate to each other. REMPLI PLC network can provide to applications (such as remote meter reading, remote device control, ...) with QoS (Quality of Service) guarantees in terms of the user data transfer needs such as reliability and delay (real-time).

How REMPLI PLC network can overcome the powerline disadvantage and designing network protocol implements QoS handling mechanisms for dynamically adapting the powerline circumstances and shortening the transmission time under stringent bandwidth limitation is presented in the next section.

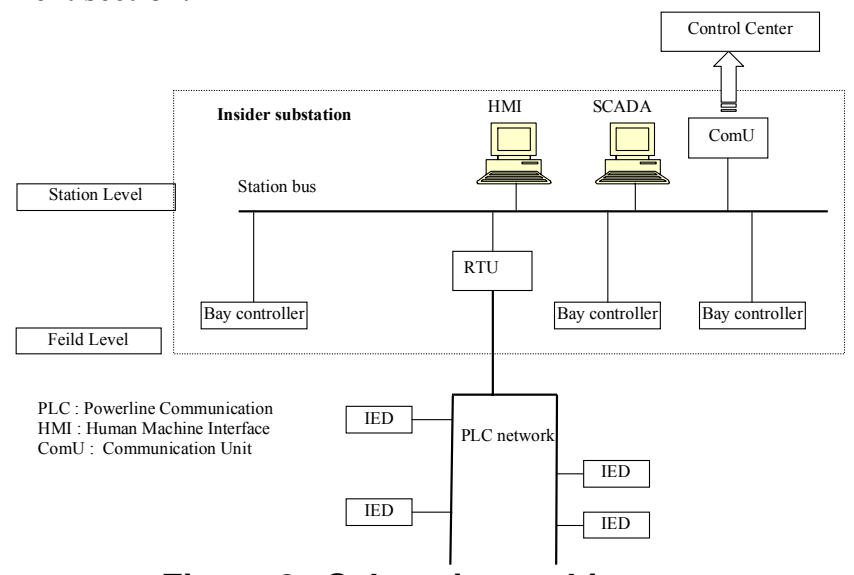

Figure 2. Substation architecture

\section{REMPLI protocols}

The protocol stack of the REMPLI PLC network system is presented in Figure 3. The powerline channel characteristics are considered to design the protocols.

For digital data transmissions over powerlines, orthogonal frequency division multiplexing (OFDM) in combination with forward error correction (FEC) can be an appropriate modulation scheme for the physical layer, which provides optimum performance if the whole available channel bandwidth is allocated to only one communication link. And a new network layer protocol has been developed, called Simple Frequency Network (SFN) [5]. It provides routing methods for the wide area PLC network. QoS mechanisms are used in the network layer for guaranteeing minimum bandwidth utilization through periodic traffic and short end- to-end delay of aperiodic data request services to satisfy the application QoS requirements. The transport layer manages all end-to-end communication, eventually using REMPLI Bridges to communicate between the MV and LV segments.

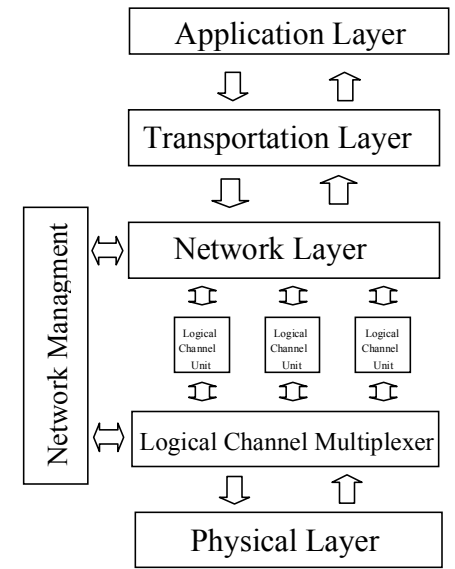

Figure 3. Protocol stack

\subsection{Logical channel model}

As OFDM systems transmit the information in parallel, the demodulation cannot start until the total symbol has been received. Therefore, the repetition cannot start immediately after the message is received. For effective utilization of PLC limited bandwidth, logical channel concept is used.

In the Time Division Multiple Access (TDMA) scheme the time axis is divided into time slots $\left(T_{\text {Symbol }}\right)$, pre-assigned to the different users. Since the whole system is based on a master-slave communication mode, the master station can provide independent logical channels in different timeslots which allows an efficient solution for the computation time problem of the stations. The logical channel division takes places in a chronological way. The slot assignments follow a predetermined pattern that repeats itself periodically; each such period is called a cycle which can be taken for a duration of logical channel.

In Figure 4, we see an example for 3 logical channels A, $\mathrm{B}$ and $\mathrm{C}$. Only one symbol is sent to the powerline within a time slot of $T_{\text {Symbol }}$. The spare time between two symbols on the powerline depends on the synchronization. The scheduling of demodulation, higher network layer processes and modulation again has an adequate time to carry out by the DSP for the physical layer and HyNet 32XS for the network layer, if 2 timeslots are between receiving and transmitting slots.

From the time axis view, the network bandwidth can be divided into the $n \quad(n \geq 3)$ channels, and each channel transmission speed rate is equal to the total speed rate divided by the number of the logical channels. In each logical channel, the network management has time to do their tasks and is able to answer or repeat the symbol directly in the next timeslot of the logical channel.

\footnotetext{
${ }^{1}$ This work has been carried out as part of the REMPLI (Real-time Energy Management via Power Line and Internet) project (European program NNE5-2001-00825, www.rempli.org)
} 


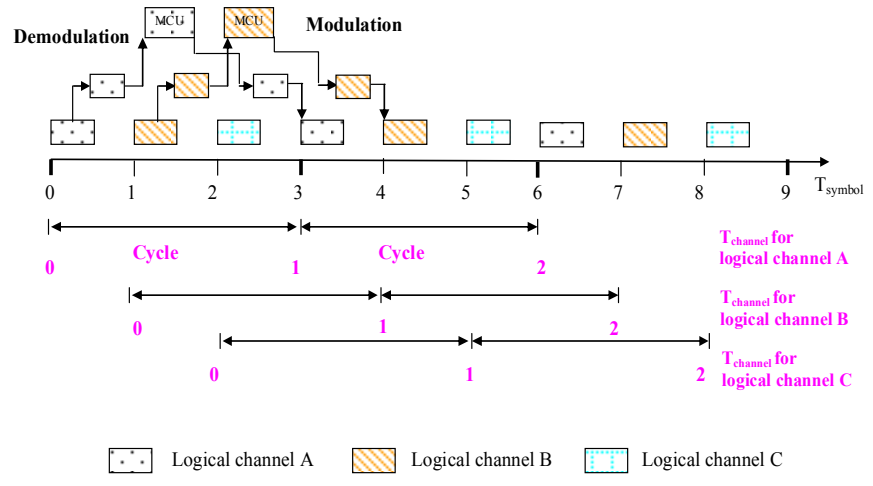

Figure 4. Depiction of the logical channels

The logical channels are used independently in the network layer. The different logical channels can be allocated to different medium access methods, which are managed by the independent logical channel unit. For example, we use 2 logical channels for a master-slave system with cyclic polling and the third timeslot as slotted aloha for very fast messages from slave to master. So different types of communication systems can be integrated and work in parallel.

Also it is possible to use the logical channels for different masters, which have to be synchronized (e.g. GPS). The slave is able to communicate with both in parallel. The system redundancy for safety applications is very high. A slave can communicate with a logical channel. It is not necessary that a slave of the network is connected to all logical channels.

For using the logical channel mode, a new installed slave requires to scan different frequency bands and transmission modes to find a used channel. After the frequency band and transmission mode is detected a synchronization to the time frame and identification of the protocol type is required.

\subsection{Network layer}

\subsubsection{Medium access}

Medium Access is controlled by Master station, normally location in the substation. The other station are polling and supervised by the master station. Polling use a priority-based scheme. Priority assignment and the bandwidth allocation are done by the dispatch in the network layer which is explained in the following section.

\subsubsection{Routing}

In a wide area PLC network, the transmission requires repeaters to relay a packet for reaching the destination node. As the dynamic topology change and impossible prediction of the powerline attenuation, repeaters must be dynamic configured.

In SFN, all slaves can work as repeaters for avoiding statically configured repeaters. The single frequency network allows several transmitters at various locations to transmit identical information on the same frequencies of the powerline medium at the same time. The receivers can get the information from the superposition of the signals [5].

The slaves, which received the initially transmitted data packet correctly by checking CRC (cyclic redundancy check), can be repeaters. In the next timeslot, the slaves send this packet to the powerline. With a flooding-based way, the data can reach the destination node without any routing table. It is the SFN routing principle. The procedure is show in Figure 5.
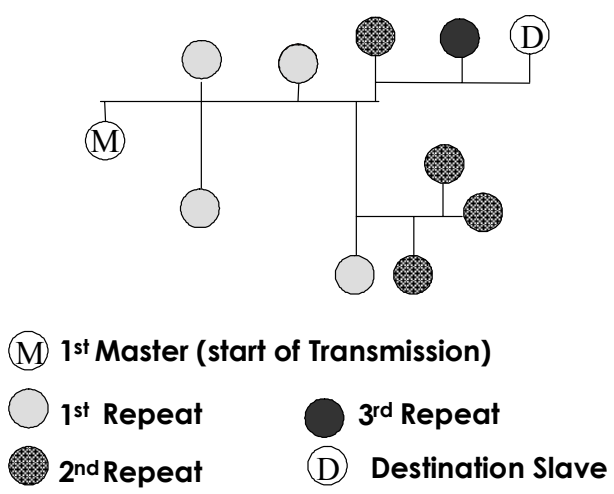

Figure 5. SFN routing principle

\subsubsection{Packet format}

The network packet format is defined as the Figure 6. For limiting the repeater time, remain repeater levels is introduced. When a packet is received correctly, the slave checks the remaining repeater level field of packet header, firstly. Then it stops working as a repeater in the following case:

--the destination address is its own address

--the remaining repeater level is zero

--the same packet has been repeated once.

Otherwise it continues to repeat this packet after remain repeater level is deceased one. When the repeater level value is equal to zero, the transmission is considered as finish.

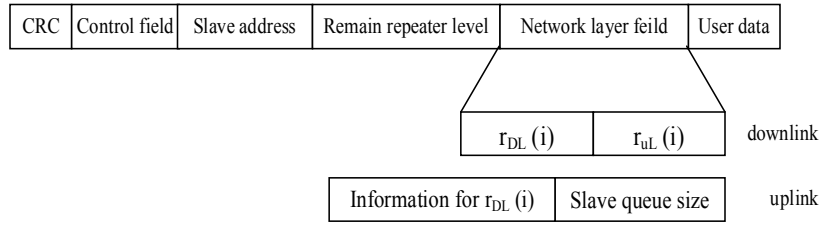

\section{Figure 6. SFN packet format}

Different repeater levels may be used in the downlink (from master to slave) and the uplink (from slave to master), considering the powerline's random channel characteristics. The table of repeater level (represented as $r_{D L}(i)$ and $r_{U L}(i)$ for downlink and uplink) for reaching every slave $i$ is stored in the master. The master indicates the downlink and uplink levels values in the network layer field at the beginning of transmission. The slave copies the allowed number of repeater levels of uplink as start value to the field 'remaining number of repeater levels' for the uplink transmission.

When a master don't receive the slave i confirmation 
within the maximum transfer time, a retransmission is required. The fail transmission is considered to be caused by the lacking repeater levels. So the master has to do:

$$
\begin{aligned}
& r_{D L}(i)=r_{D L}(i)+1 \\
& r_{U L}(i)=r_{U L}(i)+1
\end{aligned}
$$

If the retransmission was not successful with new repeater levels, the master does (1) again and sends a next retransmission. This continues until:

--Successful transmission

--Maximum number of retries

--Upper bound for downlink repeater level and uplink repeater level reached.

For maximum the network performance and shortening the transmission time, the repeater levels should be configured to the suitable values to match to the real situation. In case of an overestimation of the repeater level, the protocol acts with more precaution to decrease the repeater levels for avoiding the retry. The downlink and uplink repeater levels should be treated separately. The protocol uses the uplink network layer field information to know whether the current downlink repeater level are greater than the actual number. For the uplink network layer field can provides following information:

--Slave received a downlink packet with 'remaining number of repeater levels' $>0$

--Slave received in this logical channel during the last $r_{D L}(i)+r_{D L}(i)$ slots a downlink packet

The same information can be collected by the master. With those information, the decrease of repeater levels can be happened in two cases.

One case is that the retry leads that the downlink repeater level or uplink repeater level is more than the exactly needed. If the destination slave i received a downlink packet in this logical channel during the last $r_{D L}(i)+r_{D L}(i)$. it means that last transmission failure happened in uplink. When the master gets this information from the uplink network layer field, then $r_{D L}(i)=r_{D L}(i)-1$. Otherwise, the last failure happened in downlink. So $r_{U L}(i)=r_{U L}(i)-1$.

The other case is that the transmission was successful without retry and the master received a uplink packet with 'remaining number of repeater levels' $>0$, or the uplink network layer field indicates that the slave $\mathrm{i}$ received a downlink packet with 'remaining number of repeater levels' $>0$. Decrement of the repeater level in the better PLC condition risks the retransmission which be caused by the insufficient repeater level in the other worse conditions. Moreover, the system shall react fast to changes of the channel. The fastest implementation is to count the number of continuing successful transmission $\left(c_{D L}(i)\right.$ and $\left.c_{U L}(i)\right)$ since the last time, where respective repeater levels are necessary. When this counter greater than $r_{D L}(i)+r_{D L}(i)$, the decrement is done. This counter is cleared when

--A decrement has done.

--A transmission failed.
--Receiving packet with 'remaining number of repeater levels' $=0$ happened.

\subsubsection{Dispatcher}

The Network Layer provides three priority levels: 0, 1 and 2 for aperiodic task and hard and soft levels for the periodic polling.

The aperiodic priority levels are defined next, where a lower number means a higher priority:

--Priority 0 (CRITICAL), at Master side only.

--Priority 1 (EMERGENCY), at Master and Slave sides.

--Priority 2 (NORMAL), at Master and Slave sides.

For periodic task, two types of periodic polling level are defined: hard periodic and soft periodic. By hard periodic polling we refer to periodic polling that has stricter constraints relatively to the time period. Soft periodic polling adds a certain timing relaxations relatively to hard periodic polling.

The order of completing the traffic task is shown in Figure 6. A Round-Robin mechanism exists between aperiodic packets of priority 0 and hard periodic packets. This allows to maintain a correct management of the network (through the hard periodic packets) for one part, and to allow critical aperiodic packets to immediately be transmitted for another part; without creating network monopolization by any of them. The dashed arrow represents the promotion of periodic packets from soft to hard periodicity constraints.

Afterwards the dispatcher verifies the existence of aperiodic packets of priority 1 , followed by soft periodic packets and finally aperiodic packets of priority 2 . This order allows aperiodic packets of priority 1 to have a higher priority than soft periodic packets, since these last have lower periodic constraints. Nevertheless, the soft periodic packet can be promoted into a hard periodic packet in order to guarantee the completion of the current activation by dual-priority dispatcher with deadline relaxation [6].

In dual-priority policy, periodic packets possess two levels of priority: low and high level, whilst aperiodic packets are scheduled using a medium priority level. According to this, periodic packets can run immediately at a low level while there is no aperiodic traffic. With the aperiodic traffic, a soft periodic task should only be sent when promoted to the hard periodic, as late as possible.

\subsection{Transport layer}

The Transport layer provides reliable end-to-end data transport. It also manages the dynamics of the communication paths. Within the REMPLI Bridges, the Transport layer handles the information exchange between the MV and LV PLC networks. When there are multiple logical channels, the Transport layer is also a De/Multiplexer for different Network Layers. 


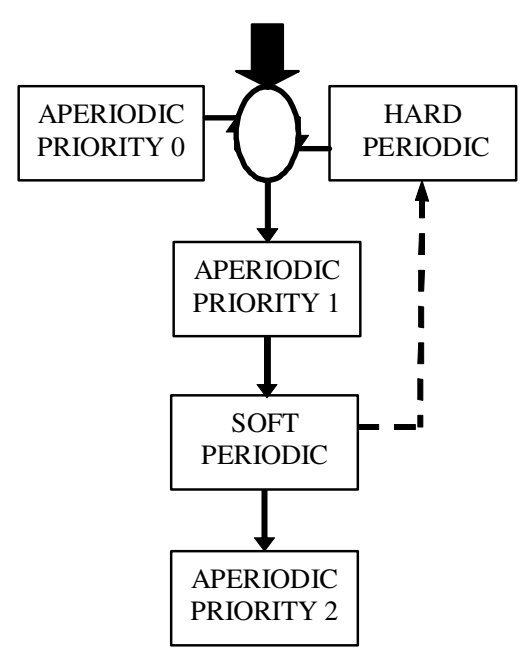

\section{Figure 7. Representation of the process of the different types of traffic}

\section{Performance evaluation by simulations}

Before we implement the whole network protocols stacks, we do the simulation to evaluate the system performance. The physical layer of powerline communication system is emulated by the Physical Layer Emulator [4] developed by iAd. The MV power grid shows a ring or radial topology. And the size of communication network for outsider substation automation depends on MV feeder equipments and its control domain. The maximum node of the communication network is not over 200. So Physical Layer Emulator provides 4 channel models which are Ring_10, Ring_100, Rand_Area Np_100, Rand_Area Np_200, for representing typical network topologies. The two former channel models have the topology of ring and the latter two have the topology of a tree with the master as the root and the randomly distributed slaves as leaves. The number in the channel model name indicates the number of the nodes. The upper layer is simulated in OPNET simulation tool.

The first scenario is to focus the protocol performance on three metrics: average retransmission per polling cycle, average duration of a polling cycle and the transmission time range in each channel models. The first metric is to evaluate the protocol stability, and the second metric is defined as the time with which the master polls once all the slaves. The last one gives the best case and the worst case of the transmission time from the master to a slave. The best case is that the slave can be reach by the master directly and the transmission error rate is zero. Whereas, the worst base is that the slave need the most repeater number to be communicated with the master and the transmission is successful after one try. Herein, we do not consider that there is more than one task in the master, since this result is difficult to give out without traffic model. Moreover, the command packets for which implement functions in Table I should be given highest priories. So we can ignore the time for waiting the channel free and take the transmission time as the performance metrics. We assume that the system has three logical channels, only one of which is used for transmitting user data. The formula to calculate the polling cycle time is given in the following.

$D=\sum_{i=2}^{n} \sum_{j=0}^{n_{\text {retry }} i}\left(2 \cdot(j+1)+r_{D L}(i)+r_{U L}(i)\right) \cdot\left(T_{s} \cdot N_{\log _{-} \text {chan }}\right)$

Parameters:

$n \quad$ number of node (master node with $\mathrm{n}=1$ )

$T_{s} \quad$ duration of one slot for transmitting a packet

$n_{\text {retry } i} \quad$ retry number of node $\mathrm{i}$

$r_{D L}(i) \quad$ repeater level of downlink (i.e., master to slave) of node $\mathrm{i}$ for the fist transmission

$r_{U L}(i) \quad$ repeater level of uplink of node i for the first transmission

$N_{\text {log_chan }}$ number of logical channel

The transmission time from master to slave can be calculated by the following formula:

$T(i)=\left(r_{D L}(i)+n_{\text {renty }} i+\sum_{j=0}^{n_{\text {rens }} ;-1}\left(2 \cdot(j+1)+r_{D L}(i)+r_{U L}(i)\right)\right) \cdot\left(T_{s} \cdot N_{\text {log }_{-} \text {chan }}\right)+T_{s}$

Table II

Simulation result of average of packet retries per polling cycle

\begin{tabular}{ll}
\hline \hline Channel Model & $\begin{array}{l}\text { Average of packet retries } \\
\text { per polling cycle }\end{array}$ \\
\hline Ring_10 & $1,1 \%$ \\
Ring_100 & $0,4 \%$ \\
RandArea $\mathrm{Np} \_100$ & $0,5 \%$ \\
RandArea $\mathrm{Np} \_200$ & $1,1 \%$ \\
\hline \hline
\end{tabular}

Table III Average duration of SFN

\begin{tabular}{lllll}
\hline \hline $\begin{array}{l}\text { Channel } \\
\text { Model }\end{array}$ & $\bar{D}_{S F N, \Sigma}$ & $\begin{array}{l}\text { Timeslot } \\
\text { duration (s) }\end{array}$ & $\begin{array}{l}\text { Logical } \\
\text { channel } \\
\text { number }\end{array}$ & $\begin{array}{l}\text { Average } \\
\text { duration(s) }\end{array}$ \\
\hline Ring_10 & 30.6 & 0.009792 & 3 & 0.8989 \\
$\begin{array}{l}\text { Ring_100 } \\
\text { RandArea }\end{array}$ & 419.9 & 0.009792 & 3 & 12.3350 \\
$\begin{array}{l}\text { Np_100 } \\
\text { RandArea }\end{array}$ & 419.3 & 0.009792 & 3 & 12.3174 \\
Np_200 & 1033.9 & 0.009792 & 3 & 30.3718 \\
\hline \hline
\end{tabular}

Table IV

Transmission time from master to slave

\begin{tabular}{ccccc}
\hline \hline $\begin{array}{c}\text { Channel } \\
\text { Model }\end{array}$ & $\begin{array}{c}\text { Maximum } \\
\text { repeater } \\
\text { number }\end{array}$ & $\begin{array}{c}\text { Minimum } \\
\text { repeater } \\
\text { number }\end{array}$ & $\begin{array}{c}\text { Maximum } \\
\text { transmission } \\
\text { time (s) }\end{array}$ & $\begin{array}{c}\text { Minimum } \\
\text { transmission } \\
\text { time (s) }\end{array}$ \\
\hline Ring_10 & 2 & 0 & 0.186048 & 0.009792 \\
Ring_100 & 3 & 0 & 0.274176 & 0.009792 \\
RandArea & 4 & 0 & 0.274176 & 0.009792 \\
Np_100 & 6 & 0 & 0.362304 & 0.009792 \\
$\begin{array}{c}\text { RandArea } \\
\text { Np_200 }\end{array}$ & 6 & & & \\
\hline \hline
\end{tabular}

In Table II, the simulation results show the network layer protocol has small retransmission time percent $(<1.2 \%)$. Table III shows that the average duration is small, even 30.3718 seconds for polling 199 slaves within one of three 
logical channels. So the network layer protocol can provide a short transmission time and few retransmissions in the powerline environment [7].

In Table IV, the minimum transmission times of all channel models are less than $10 \mathrm{~ms}$. It can satisfy the communication performance constrains of the Table I. Unfortunately, the maximum transmission times are greater than $10 \mathrm{~ms}$, but less than $1 \mathrm{~s}$. So the network requirement of most outsider substation applications can be guaranteed.

In the first simulation scenario, the master only implements polling tasks. If PLC communication system adopts periodic polling as a transmission mode, the medium access delay may be equal to a polling cycle in the worst case. The application time constraints cannot be satisfied. So we introduce priorities and dispatching function in the network layer which can schedule different priorities for guaranteeing a certain QoS to the important application data, as well as ensuring a stable network management system.

We build another simulation scenario to evaluate the access delay of aperiodic packets when the system has periodic and aperiodic task with different priorities. The access delay of an aperiodic packet is the time between being available in the queue and sending into the PLC network. It permits to evaluate the delay of the aperiodic packets, at the master side.

The simulation is done in Ring_10 channel model. The master sends aperiodic packets to all slaves in a uniform manner, but always respecting the following periodic traffic:

--P0 : generates a polling task for slave 1 per every 255 timeslots, the deadline equaling to the period and has the soft periodic level

--Pa : generates a polling cycle per every 3840 timeslots, the deadline equaling to the period and has the hard periodic level

$--\mathrm{Pb}$ : generates a polling cycle per every 378 timeslots, the deadline equaling to the period and has the soft periodic level

In the scenario of a single aperiodic queue, we use a single aperiodic queue of priority 2 (normal), with a buffer size of 40 packets. In the scenario of two aperiodic queues there are Aperiodic Priority 1 and Aperiodic Priority 2 queue with each queue buffer size of 20 packets. Aperiodic packets in the first queue (Aperiodic Priority 1) have a higher priority than soft periodic packets. However, in the case of missing deadlines, these soft periodic packets are promoted to the hard periodic table, with a higher priority than any aperiodic queue, which allows not missing the deadline again.

Figure 8 shows the percentage acceptance of aperiodic packets with the coordination of the packet generation rate. Because some packet can be dropped in consequence of a queue buffer overflow condition, the percentage is low when the traffics are heavy. And the higher priority queues suffer lightly than the lower priority queues due to the dispatcher scheduling [8].

In Figure 9, the introduction of the promotion from soft to hard periodic level shows that with a high load (aperiodic packets generated every 5 to 8 timeslots), the aperiodic priority 2 suffers greatly, while aperiodic priority 1 still maintains a small access delay, the maximum value of which is 19 timeslots $(0.0128 \mathrm{~s})$. Adding this value to the maximum transmission time, we get new values which are still less than $1 \mathrm{~s}$.

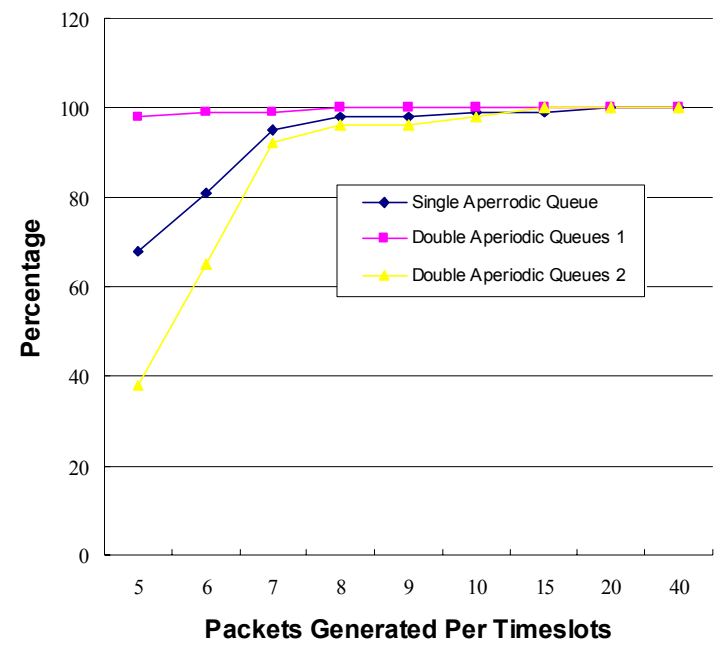

Figure 8. Percentage acceptance of aperiodic packets

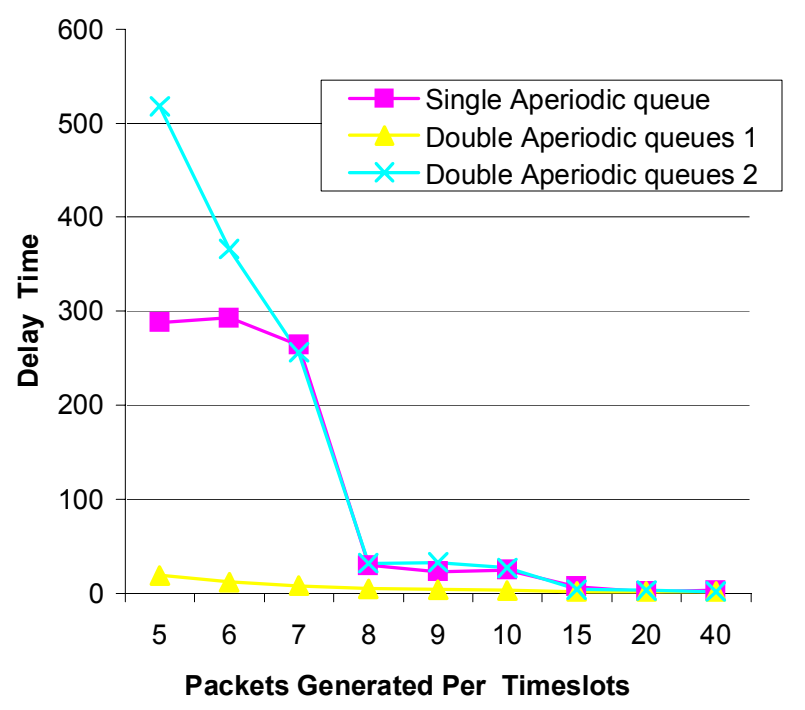

Figure 9. Delay times of aperiodic packets generated at several timeslot units' rates

\section{Conclusion}

In this paper, we propose using REMPLI communication system to connect the outsider substation feeder equipment for substation automation. REMPLI network protocols are designed for the PLC. Before implementation of the communication protocol on the embedded system, we do the simulation to evaluate the protocol performance. Our simulation results have shown that our new network protocol can guarantee minimum bandwidth utilization 
through periodic traffic and short end-to-end delay of aperiodic data request services. The most communication requirement of substation automation application can be satisfied in our REMPLI PLC network. For the small transmission time requirements which are tens milliseconds, they can be satisfied when the feeder devices locations are near the substation. In the future, the small transmission time requirements will be satisfied with smaller timeslot duration, which will be carried out by higher speed network interface card under development within REMPLI European project.

\section{Acknowledgement}

The authors are grateful to Raul Brito for doing part of simulations and the dispatcher implementation, as well as Gerd Bumiller for providing the SFN and part of REMPLI network layer protocol description.

\section{References}

[1] Report, "The Automation of New and Existing Substations: Why and How", Sponsored by the CIGRE Study Committee B5, August 2003

[2] IEEE 2003, March. Draft IEEE SA technical report on substation integrated protection, control and data acquisition communication requirements. Technical Report IEEETR 1525-2003, Substation Committee of the IEEE Power Engineering Society

[3] C.R.Ozansoy, A.Zayegh, A.Kalam, "Communications for Substation Automation and Integration", In Australasian Universities Power Engineering Conference, Available at: http://www.itee.uq.edu.au/ aupec/aupec02/Final-Papers/C-ROzansoy1.pdf

[4] Gerd Bumiller, "Power-Line Physical Layer Emulator for Protocol Development", In 8th International Symposium on Power-Line Communications and its applications.

[5] Gerd Bumiller, "Single Frequency Network Technology for Medium Access and Network Management", In 6th International Symposium on Power-Line Communications and its application.

[6] Raul Brito and Yeqiong Song, "A Dispatching Mechanism Providing REMPLI Applications with QoS", In 10th IEEE International Conference on Emerging Technologies and Factory Automation.

[7] Gerd Bumiller, Liping Lu, Yeqiong Song, "Analytic Performance Comparison of Routing Protocols in MasterSlave PLC Networks", In 9th International Symposium on Power-Line Communications and Its Applications.

[8] Liping Lu, Raul Brito, Yeqiong Song, "QoS and Performance of REMPLI PLC Network", In 1st Workshop on Networked Control System and Fault Tolerant Control - NeCST Workshop 2005. 